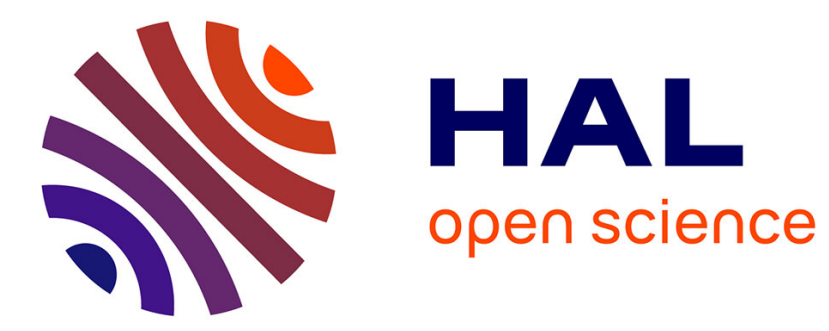

\title{
Effective mechanical response of non-linear heterogeneous materials comprising bimodular phases
} Elisabetta Monaldo, Stella Brach, Djimedo Kondo, Giuseppe Vairo

\section{To cite this version:}

Elisabetta Monaldo, Stella Brach, Djimedo Kondo, Giuseppe Vairo. Effective mechanical response of non-linear heterogeneous materials comprising bimodular phases. European Journal of Mechanics A/Solids, 2020, 81, pp.103962. 10.1016/j.euromechsol.2020.103962 . hal-02484203

\section{HAL Id: hal-02484203 https://hal.science/hal-02484203}

Submitted on 19 Feb 2020

HAL is a multi-disciplinary open access archive for the deposit and dissemination of scientific research documents, whether they are published or not. The documents may come from teaching and research institutions in France or abroad, or from public or private research centers.
L'archive ouverte pluridisciplinaire HAL, est destinée au dépôt et à la diffusion de documents scientifiques de niveau recherche, publiés ou non, émanant des établissements d'enseignement et de recherche français ou étrangers, des laboratoires publics ou privés. 


\title{
Effective mechanical response of non-linear heterogeneous materials comprising bimodular phases
}

\author{
Elisabetta Monaldo ${ }^{\mathrm{a}, *}$, Stella Brach ${ }^{\mathrm{b}}$, Djimédo Kondo $^{\mathrm{c}}$, Giuseppe Vairo $^{\mathrm{d}}$ \\ ${ }^{a}$ University of Rome "Niccolò Cusano", Rome, Italy \\ ${ }^{b}$ LMS, CNRS, Ecole Polytechnique, Institute Polytechnique de Paris, Palaiseau, 91128, \\ France \\ ${ }^{c}$ Institut Jean le Rond D'Alembert, Sorbonne Université, Paris, France. \\ ${ }^{d}$ Department of Civil Engineering and Computer Science (DICII), University of Rome "Tor \\ Vergata", Rome, Italy
}

\begin{abstract}
In this study, the effective constitutive behavior of heterogeneous materials comprising bimodular phases is investigated through a computational approach and by referring to Curnier-type bimodularity. Different microstructures characterized by spherical inclusions or voids are addressed, by analyzing different loading scenarios. Numerical results, obtained via an iterative finite-element scheme, highlight the influence of intraphase constitutive non-linearities induced by the tension/compression transition of the local material stiffness. Moreover, coupling effects between hydrostatic and deviatoric states are elucidated. The macroscale material response results dependent on the loading condition, and it is driven by perturbative effects of heterogeneous fields locally induced by pores or inclusions.
\end{abstract}

Keywords: bimodular composites, bimodular porous materials, non-linear constitutive response, computational homogenization.

\section{Introduction}

Creating materials with optimized overall properties has long been a central concern in many engineering applications. As such, a variety of heterogeneous materials (e.g., composites, porous media) have been manufactured in the past, by designing specific microstructures in order to satisfy specific targets. However, for some of those materials, the relation between microscopic physical mechanisms (arising at the local lengthscale of the heterogeneities) and the

\footnotetext{
* Corresponding author

Email addresses: elisabetta.monaldo@unicusano.it (Elisabetta Monaldo), stella.brach@polytechnique.edu (Stella Brach), djimedo.kondo@upmc.fr (Djimédo Kondo), vairo@ing.uniroma2.it (Giuseppe Vairo)
} 
macroscopic constitutive response (observed at the larger scale of the specimen) has yet to be completely identified.

This is for instance the case of bimodular materials, defined as elastic materials exhibiting different costitutive response in tension and in compression (?????). A wide number of materials belong to such a class of macroscale nonlinear constitutive behavior, as for instance concrete, biological gels, graphite, NiTi shape memory alloys, fiber-reinforced composite materials, damaged materials containing microcracks (?????).

In the available literature, bimodularity effects have been generally explored by referring to classes of engineering-relevant materials whose constitutive response is bimodular at the macroscopic lengthscale, even though their subconstituents locally exhibit a linear elastic behavior. This is for instance the case of layered materials such as nacre (?) or fiber-reinforced composites (?), whose effective elastic response results bimodular as a consequence of interphases nonlinearity sources. These latter are for instance related to the unilateral contact between microstructural domains (?), unilateral response of damaged interfaces between different phases (???), microscale instabilities (?), asymmetric mechanical response of microvoids or microcracks (??). Many recent studies address such a non-linear mechanical response via both experimental and analytical approaches(??????????).

At the macroscale, the constitutive response of these materials is described by discriminating the tension/compression values of the elastic moduli in terms of some relevant stress or strain measures (?). A modeling approach is based on the criterion of positive-negative signs of principal stresses, proposed by Ambartsumyan (?) and mainly applied for describing bimodular materials with an isotropic macroscale symmetry (????????). Some macroscopic fiber-governed models, inspired to the pioneering work of ?, refer to the sign of the strain component along the fiber direction. Many applications of such an approach to hierarchical fiber-reinforced composites can be found in literature (??????????). A consistent generalization in the framework of the so-called conewise materials was proposed by ?, by extending basic elements of classical smooth elasticity to piecewise smooth elasticity under the continuity requirement of strain energy and stress (?).

Notwithstanding the effort provided in theoretical modelling, and also due to the variety of materials involved, a complete characterization of the elastic response of bimodular media has not been furnished yet. The main open questions are the following. It is not clear whether a material with bimodular constituents also behaves as bimodular at the macroscopic scale. Further, it is not fully understood how the non-linearity induced by heterogeneous strain fields impacts on the overall response of the material.

This paper aims to contribute to answer to those questions. Different composite and porous materials, where one or more of the constituents are bimodular, are numerically investigated. In detail, reference is made to the elastic bimodularity traced by ? and briefly recalled in Section 2 2 The numerical treatment of the non-linear elastic microscale problem introduced in Section 2 is discussed in Section 3, specifically addressing different microstructures and 
loading scenarios. Results, obtained via non-linear computations based on an iterative finite-element scheme, are proposed in Section 4. Finally, some concluding remarks are summarized in Section 5 .

\section{Problem statement}

Let $\Omega$ be a representative volume element (rve) of a composite material, whose exterior boundary is $\partial \Omega$, consisting of a matrix $\left(\Omega^{1}\right)$ and a spherical inclusion $\left(\Omega^{2}\right)$ with volume fraction $f=\left|\Omega^{2}\right| /|\Omega|$ (see Fig.1). The domain is a cube of edge length $B$ and the inclusion is centered. The same rve is used to study a porous material, while intending $\Omega^{2}$ as a void region and $f$ as the porosity $p$. The following notation is adopted throughout the paper: bold characters denote vectors and second-order tensors; blackboard letters indicate fourth-order tensors; the symbol : stands for the double product operator; $\mathbb{I}=$ $(\mathbf{I} \otimes \mathbf{I}) / 3$ and $\mathbb{K}=\mathbb{I}-\mathbb{J}$ are the hydrostatic and deviatoric fourth-order projector tensors ( $\mathbb{I}$ being the fourth-order identity tensor), respectively, and the volume average of space-dependent quantities over the domain $\Omega$ is defined as

$$
\langle\cdot\rangle=\frac{1}{|\Omega|} \int_{\Omega} \cdot d \Omega .
$$

A Cartesian reference system is introduced with respect to which $\boldsymbol{x}$ identifies the position of a point in $\Omega$. Microscopic (resp., macroscopic) second-order strain and stress tensors are $\boldsymbol{\varepsilon}(\boldsymbol{x})$ and $\boldsymbol{\sigma}(\boldsymbol{x})$ (resp., $\mathbf{E}$ and $\boldsymbol{\Sigma}$ ), whereas $\boldsymbol{\xi}(\boldsymbol{x})$ is the microscopic displacement field.

Phases are considered as perfectly bonded and the rve is assumed to undergo homogeneous strain boundary conditions. As a result, the elastic microscale problem is:

$$
\begin{aligned}
& \left\{\begin{array}{l}
\operatorname{div} \boldsymbol{\sigma}(\boldsymbol{x})=\mathbf{0} \\
\boldsymbol{\sigma}(\boldsymbol{x})=\mathbb{C}(\boldsymbol{x}, \boldsymbol{\varepsilon}(\boldsymbol{x})): \boldsymbol{\varepsilon}(\boldsymbol{x}) \quad \boldsymbol{x} \in \Omega \\
\boldsymbol{\varepsilon}(\boldsymbol{x})=\operatorname{sym} \nabla \boldsymbol{\xi}(\boldsymbol{x})
\end{array}\right. \\
& \boldsymbol{\xi}(\boldsymbol{x})=\mathbf{E} \cdot \boldsymbol{x} \quad \boldsymbol{x} \in \partial \Omega
\end{aligned}
$$

where the affine displacement conditions in Eq. (3) comply with the average rule $\mathbf{E}=\langle\varepsilon\rangle$. The macroscopic second-order stress tensor $\boldsymbol{\Sigma}$ is defined by the stress average: $\boldsymbol{\Sigma}=\langle\boldsymbol{\sigma}\rangle$.

Constitutive response of each phase is assumed to obey to an elastic behavior, generally non-linear. In detail, the stress-strain relationship is $\boldsymbol{\sigma}(\boldsymbol{x})=$ $\mathbb{C}(\boldsymbol{x}, \boldsymbol{\varepsilon}(\boldsymbol{x})): \boldsymbol{\varepsilon}(\boldsymbol{x})$, where the secant elasticity tensor $\mathbb{C}$ generally depends on the position $\boldsymbol{x}$ and on the local strain state $\boldsymbol{\varepsilon}(\boldsymbol{x})$. Aiming to investigate the nonlinear mechanical response of composite and porous materials comprising at least a bimodular phase, two classes of constitutive descriptions are considered: an isotropic linearly elastic constitutive law ( $\mathbb{C}$ constant with respect to $\boldsymbol{\varepsilon}(\boldsymbol{x})$ ), and an isotropic Curnier-type piece-wise linear elastic behavior, characterized by $\mathbb{C}$ locally depending on $\boldsymbol{\varepsilon}(\boldsymbol{x})$. In the second case, the elastic moduli of the 


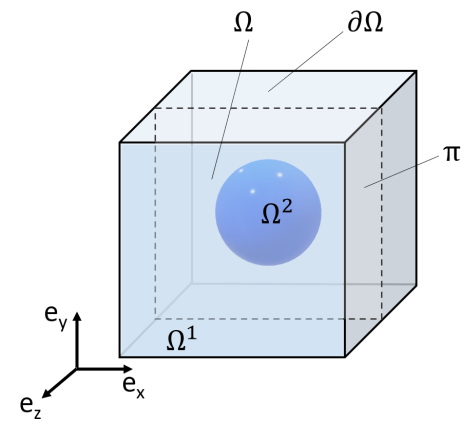

Figure 1: Representative volume element. Notation.

material vary in each point to accommodate the evolution of the strain state and, as briefly recalled in the Appendix, the elasticity tensor results in (?):

$$
\mathbb{C}(\varepsilon)= \begin{cases}\mathbb{C}_{-}=2 \mu \mathbb{K}+3 k_{-} \mathbb{J} & \text { if } \operatorname{tr}(\boldsymbol{\varepsilon})<0 \\ \mathbb{C}_{+}=2 \mu \mathbb{K}+3 k_{+} \mathbb{J} & \text { if } \operatorname{tr}(\boldsymbol{\varepsilon})>0\end{cases}
$$

where $\mathbb{C}_{-}$and $\mathbb{C}_{+}$identify compression-like and tensile-like elasticity tensors, respectively; $k_{-} / k_{+}$are the compression/tension values of the bulk modulus, and $\mu$ is the shear modulus. Note that the bimodularity only manifests itself via the volumetric part of the strain energy, whereas the deviatoric one is unaffected. Equation (4) leaves undefined the case $\operatorname{tr}(\varepsilon)=0$ (namely, corresponding to neutral strain states) where the bulk modulus discontinuously jumps from $k_{-}$to $k_{+}$. Strain states corresponding to null volumetric part are numerically handled as described in the Section 3

\section{Numerical modelling}

Different rves with a single pore or with a single inclusion are defined by considering two values of volume fraction/porosity (5\% and 15\%), and they are compared with the case of a bulk material $(f=p=0)$. Various study cases are addressed, where either one or both the constituents are assumed as bimodular. Table 1 reports the adopted notation and material properties.

Representative volume elements with randomly-distributed inclusions are considered. In this case, inclusions are linearly elastic and embedded in a piecewise linear matrix with volume fraction $f=5 \%$ (i.e., study case PL1_L2 in Table 11. A cubic domain with 27 identical inclusions, and a cubic domain with 64 inclusions, characterized by different sizes (ratio between maximum and minimum diameters equal to 4) are defined (see Fig. 22). In the first case, the minimum distance among inclusions centers, normalized with respect to $B$, results equal to 0.26 (normalized standard deviation equal to 0.04 ). In the second case, the minimum distance among inclusions centers, normalized with 


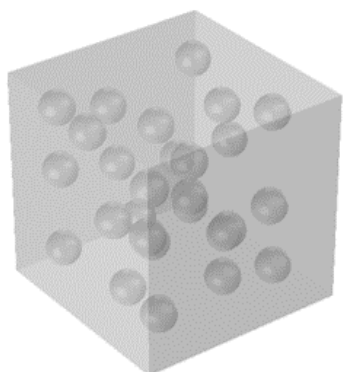

(a)

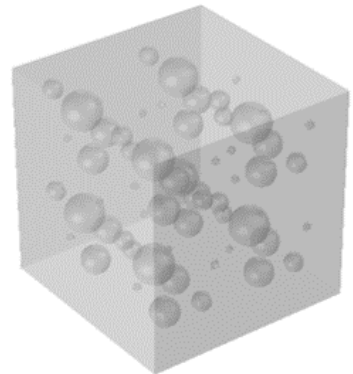

(b)

Figure 2: Representative volume elements with randomly-distributed inclusions. (a) cubic domain with 27 identical inclusions; (b) cubic domain with 64 inclusions, characterized by different sizes (ratio between maximum and minimum diameters equal to 4 ).

respect to $B$, results equal to 0.2 (normalized standard deviation equal to 0.06 ), and the normalized average radius of inclusions is 0.047 (normalized standard deviation equal to 0.025). For both multi-inclusion rve models, inclusions do not touch or intersect each other.

Each three-dimensional rve model is discretized via 10-nodes Lagrange tetrahedral elements, with second-order interpolating functions. The average mesh size is set equal to $5 \cdot 10^{-2} B$ (resp., $2 \cdot 10^{-2} B$ ) for single-inclusion domains (resp., multi-inclusion domains). Various homogeneous strain boundary conditions are enforced: uniaxial (UNI), biaxial (BIA), hydrostatic (HYD), purely deviatoric $(\mathrm{DEV})$ and mixed (HYD+DEV) loadings. With reference to the notation introduced in Eq. (3) and in Fig. 1, the macroscopic strain tensor is assigned as:

$$
\mathbf{E}= \begin{cases}\varepsilon_{0} \mathbf{e}_{\mathrm{x}} \otimes \mathbf{e}_{\mathrm{x}} & \text { UNI } \\ \varepsilon_{0}\left(\mathbf{e}_{\mathrm{x}} \otimes \mathbf{e}_{\mathrm{x}}+\mathbf{e}_{\mathrm{y}} \otimes \mathbf{e}_{\mathrm{y}}\right) & \text { BIA } \\ \varepsilon_{0}\left(\mathbf{e}_{\mathrm{x}} \otimes \mathbf{e}_{\mathrm{x}}+\mathbf{e}_{\mathrm{y}} \otimes \mathbf{e}_{\mathrm{y}}+\mathbf{e}_{\mathrm{z}} \otimes \mathbf{e}_{\mathrm{z}}\right) & \text { HYD } \\ \gamma_{0}\left(\mathbf{e}_{\mathrm{x}} \otimes \mathbf{e}_{\mathrm{y}}+\mathbf{e}_{\mathrm{y}} \otimes \mathbf{e}_{\mathrm{x}}\right) & \text { DEV } \\ \varepsilon_{0}\left(\mathbf{e}_{\mathrm{x}} \otimes \mathbf{e}_{\mathrm{x}}+\mathbf{e}_{\mathrm{y}} \otimes \mathbf{e}_{\mathrm{y}}+\mathbf{e}_{\mathrm{z}} \otimes \mathbf{e}_{\mathrm{z}}\right)+ & \text { HYD }+\mathrm{DEV} \\ +\gamma_{0}\left(\mathbf{e}_{\mathrm{x}} \otimes \mathbf{e}_{\mathrm{y}}+\mathbf{e}_{\mathrm{y}} \otimes \mathbf{e}_{\mathrm{x}}\right) & \end{cases}
$$

where $\varepsilon_{0} \in[-0.1,0.1]$ and $\gamma_{0} \in[0,0.1]$ are dimensionless strain parameters, and symbol $\otimes$ indicates the dyadic product.

Owing to the piece-wise linearity introduced by a bimodular phase, an iterative scheme is numerically implemented (see Fig. 3p by a custom Matlab code (MathWorks Inc., Natick, MA), integrated within the finite-element environment Comsol Multiphysics (COMSOL Inc., Burlington, MA). This allows to match local values of the bulk modulus $k(\operatorname{tr} \varepsilon)$ with the sign of $\operatorname{tr} \varepsilon$. Assuming the material properties as element-wise distributed, the correspondence between the value of the local bulk modulus $k$ (i.e., $k_{-}$or $k_{+}$) and the sign of $\operatorname{tr} \varepsilon(\boldsymbol{x})$ for each element of the bimodular domain is checked (see Eq. (44)). The local elastic properties are initialized as equal to the tension ones. Afterwards, 


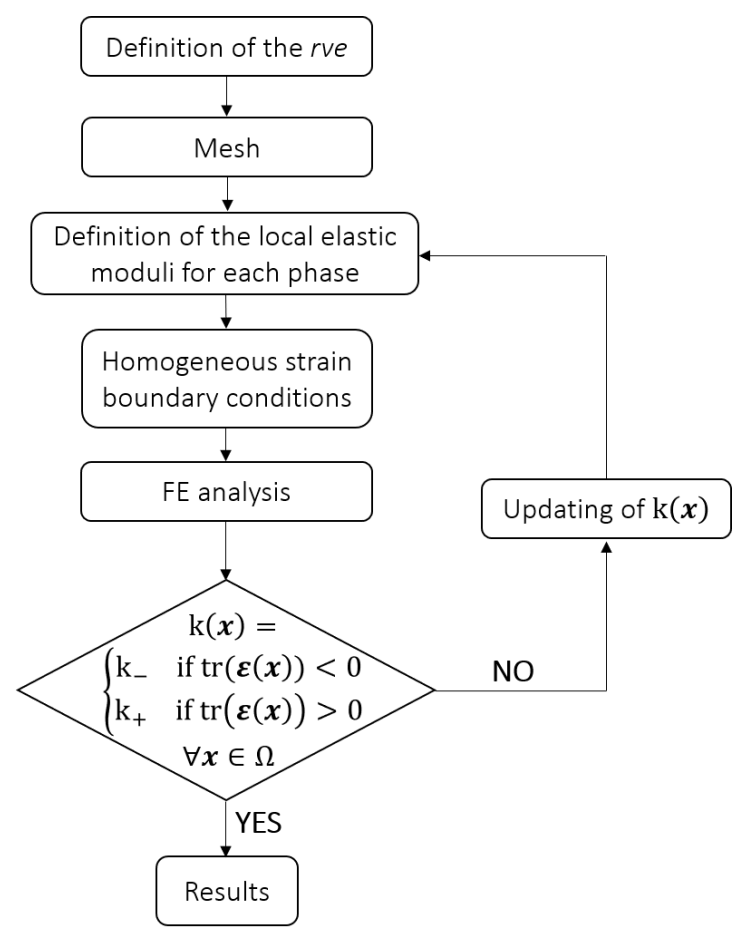

Figure 3: Solution algorithm implemented in present study.

the loading condition is applied and the microscopic strain field is computed. The element-wise value of $\operatorname{tr} \varepsilon(\boldsymbol{x})$ is computed by referring to the corresponding element orthocenter. In agreement with Eq. (4), for finite elements where $\operatorname{tr} \varepsilon(\boldsymbol{x})<0$ the local elastic properties are assigned equal to the compression ones. The computational step associated to the considered loading condition is repeated until no further changes in local elastic properties are needed. The iterative procedure converges irrespective of initialization conditions. For instance, referring to the case PL1_L2 (with $f=5 \%$ ) under a DEV loading scenario (see Table 1 and Eq. (5)), Fig. 4 depicts the number $n$ of elements where changes of the material properties occur during the simulations (normalized with respect to the total number of elements $N$ ) versus the iteration step $i$. For bimodular elements three initialization cases are investigated: with tension properties; with compression properties; random initialization. As it clearly appears, the convergence rate is practically the same, the solution reaching the convergence after five steps.

Situations though may occur where the absolute value of the hydrostatic strain is smaller than the numerical precision. In those cases, the bulk modulus of elements with $|\operatorname{tr} \varepsilon|<10^{-6}$ (physically corresponding to local neutral strain states) is assigned as follows: (i) $k_{+}$, (ii) $k_{-}$and (iii) $\left(k_{-}+k_{+}\right) / 2$. Sensitivity analyses showed that results are almost identical in the three cases. The third 
Table 1: Material properties for case studies herein considered. As a notation rule, $\mathrm{XX} r \_\mathrm{XX} r$ identifies the XX-type constitutive law (L for linearly elastic and PL for piece-wise linearly elastic) for the phase $r$ ( 1 for the matrix and 2 for the inclusion). As regards the bulk modulus, values in (respectively, not inside) brackets refer to tensile (respectively, compression) restriction.

\begin{tabular}{lccccc}
\hline & $f[\%]$ & $k^{1}[\mathrm{GPa}]$ & $\mu^{1}[\mathrm{GPa}]$ & $k^{2}[\mathrm{GPa}]$ & $\mu^{2}[\mathrm{GPa}]$ \\
\hline Porous/bulk & & & & & - \\
PL1 & $0 ; 5 ; 15$ & $2.4(20.1)$ & 1.9 & - & - \\
Composites & & & & & \\
L1_PL2 & $5 ; 15$ & 7.5 & 3.5 & $24.2(201.2)$ & 18.9 \\
PL1_L2 & $5 ; 15$ & $2.4(20.1)$ & 1.9 & 179.0 & 189.0 \\
PL1_PL2 & $5 ; 15$ & $2.3(8.4)$ & 1.7 & $24.2(201.2)$ & 18.9 \\
\hline
\end{tabular}

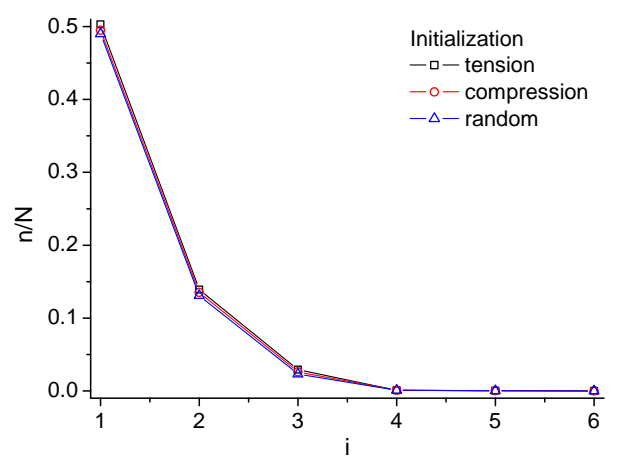

Figure 4: Convergence of the computational procedure. Number $n$ of bimodular elements where changes of the material properties occur during the simulations (normalized with respect to the total number of elements $N$ ) vs. the iteration step $i$. Case PL1_L2 (with $f=5 \%$ ) under a DEV loading condition (see Table 1 and Eq. (5)). 
Table 2: The hydrostatic, the second-order deviatoric and the third-order deviatoric macroscopic strain invariants corresponding to the macroscopic strain boundary conditions defined in Eq. (5.)

\begin{tabular}{cccccc}
\hline & UNI & BIA & HYD & DEV & HYD+DEV \\
\hline $\mathrm{I}_{1}^{\mathrm{E}}$ & $\varepsilon_{0}$ & $2 \varepsilon_{0}$ & $3 \varepsilon_{0}$ & 0 & $3 \varepsilon_{0}$ \\
$\mathrm{~J}_{2}^{\mathrm{E}}$ & $\varepsilon_{0}^{2} / 3$ & $\varepsilon_{0}^{2} / 3$ & 0 & $\gamma_{0}^{2}$ & $\gamma_{0}^{2}$ \\
$\mathrm{~J}_{3}^{\mathrm{E}}$ & $2 \varepsilon_{0}^{3} / 27$ & $-2 \varepsilon_{0}^{3} / 27$ & 0 & 0 & 0 \\
\hline
\end{tabular}

description is thus adopted in the following.

For each loading condition, the effective stress invariants are computed. The hydrostatic, the second-order deviatoric and the third-order deviatoric macroscopic stress invariants are defined as $I_{1}^{\Sigma}=\operatorname{tr} \boldsymbol{\Sigma}, J_{2}^{\Sigma}=\boldsymbol{\Sigma}_{\mathrm{dev}}: \boldsymbol{\Sigma}_{\mathrm{dev}} / 2$, and $J_{3}^{\Sigma}=\operatorname{det} \boldsymbol{\Sigma}_{\mathrm{dev}}$, where $\boldsymbol{\Sigma}_{\mathrm{dev}}$ is the deviatoric part of the macroscopic stress tensor. On the other hand, the strain invariants are $I_{1}^{\mathrm{E}}=\operatorname{tr} \mathbf{E}, J_{2}^{\mathrm{E}}=\mathbf{E}_{\mathrm{dev}}: \mathbf{E}_{\mathrm{dev}} / 2$, and $J_{3}^{\mathrm{E}}=\operatorname{det} \mathbf{E}_{\mathrm{dev}}$, where $\mathbf{E}_{\mathrm{dev}}$ is the deviatoric part of the macroscopic strain tensor. The microscopic stress and strain invariants can be similarly defined.

Table 2 summarizes the values of the macroscopic strain invariants corresponding to the boundary conditions introduced in Eq. (5).

\section{Numerical results}

In what follows, numerical results obtained by considering the previouslyintroduced procedure are presented and analyzed.

\subsection{Single-pore rves}

The macroscopic mechanical responses of single-pore rves undergoing uniaxial, biaxial, hydrostatic, and purely deviatoric loading conditions are reported in Figs. 5 and 6 , by comparing cases associated to different values of porosity $p$, including also the bulk material case $(p=0)$.

For the porous material, numerical results reveal that:

- under uniaxial, biaxial, and hydrostatic loading, the macroscopic stress invariants exhibit an asymmetric behavior with respect to the macroscopic neutral state (i.e., $\varepsilon_{0}=0$ ), as depending on the porosity $p$;

- the increase of the porosity level tends to reduce the piece-wise linearity feature in the effective hydrostatic stress response (namely, referring to $I_{1}^{\Sigma}$ ) with respect to the bulk case, mainly by reducing the slope for positive macroscale strains;

- in contrast to the bulk case, the porosity affects the macroscopic deviatoric stress response (namely described by $J_{2}^{\Sigma}$ and $J_{3}^{\Sigma}$ ) by introducing an asymmetry with respect to the macroscopic neutral state when tensile and compressive strain boundary conditions are analyzed; 
- a hydrostatic loading does not introduce any deviatoric stress state at the macroscale $\left(J_{2}^{\Sigma}=J_{3}^{\Sigma}=0\right.$ for each analyzed value of $\left.\varepsilon_{0}\right)$;

- a purely deviatoric strain boundary condition induces a coupled macroscale hydrostatic response, proportional to the porosity degree (see Fig. 6(b)) .

Hence, proposed results show that a porous bimodular material exhibits a coupling (resp., uncoupling) between hydrostatic and deviatoric macroscopic stress response when a purely deviatoric (resp., purely hydrostatic) macroscopic strain is applied.

\subsection{Single-inclusion rves}

Results concerning the macroscopic mechanical response of single-inclusion rves undergoing uniaxial, biaxial, hydrostatic, and purely deviatoric loading conditions are reported in Figs. 7 to 12 , by comparing cases associated to different values of inclusion volume fraction $f$. In particular, Figs. 7,8 and Figs. 9 10 refer to the cases characterized by the bimodularity of only one phase. Figures 7 8 address the case of a bimodular inclusion (L1_PL2, see Table 1), whereas Figs. 9 10 refer to the case of a bimodular matrix (PL1_L2). Finally, Figs. 11 12 show results for a single-inclusion rve with both matrix and inclusion characterized by a bimodular constitutive law (PL1_PL2).

For the case of a piece-wise inclusion embedded in a linear elastic matrix, proposed results show that:

- the macroscopic hydrostatic stress invariant exhibits a very slight bilinear feature, tending to increase when the volume fraction $f$ of the bimodular inclusion increases;

- independently of $f$ value, the second- and third-order deviatoric stress invariants remain symmetric in tension and compression (see Figs. 7(c) to $7(\mathrm{f})$;

- similarly to the case of a porous bimodular material, a coupling between deviatoric and hydrostatic effective responses occurs when a purely deviatoric loading is applied, as depending on the volume fraction $f$ of the bimodular phase.

As regards the case of a linear elastic inclusion embedded in a bimodular matrix:

- a bilinear response similar to the case of a bimodular porous rve is observed;

- the matrix bimodularity reflects in a strong bilinear behavior of the hydrostatic invariant at the macroscale and in an evident asymmetry of the macroscopic deviatoric stress invariants with respect to the extension and contraction states; 


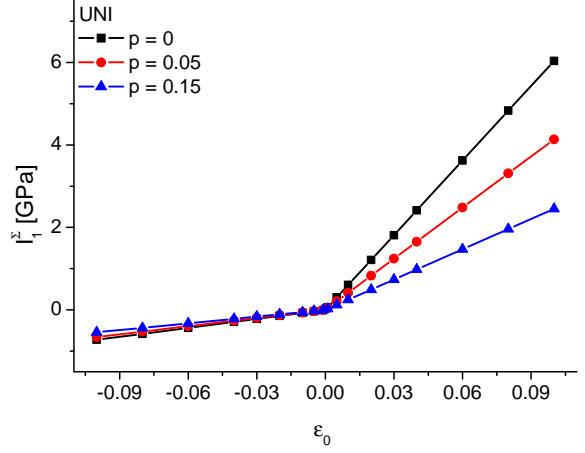

(a)

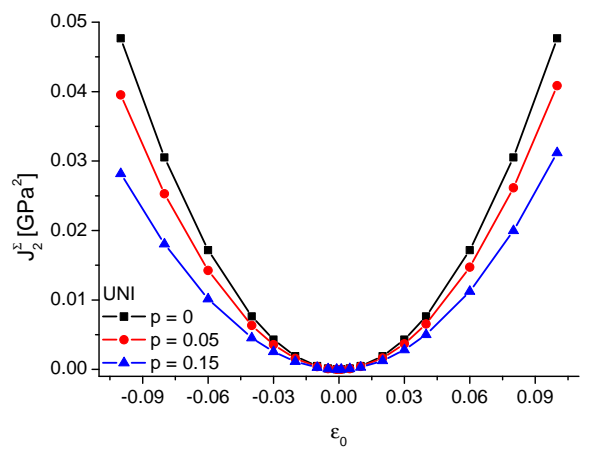

(c)

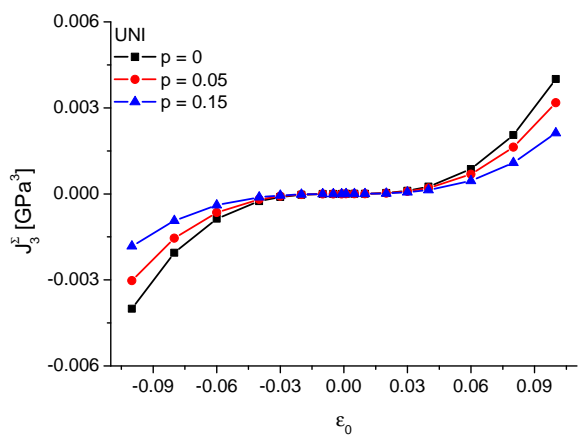

(e)

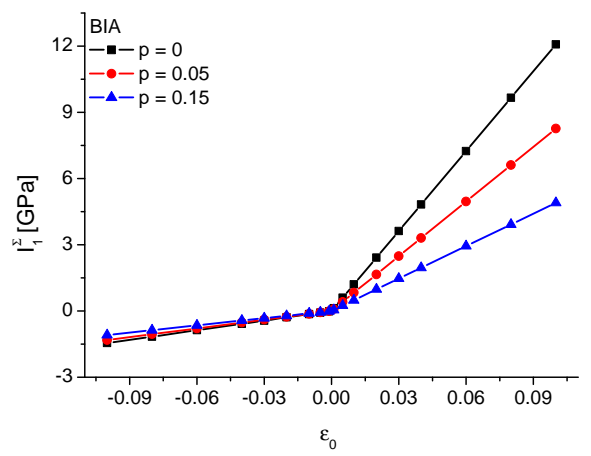

(b)

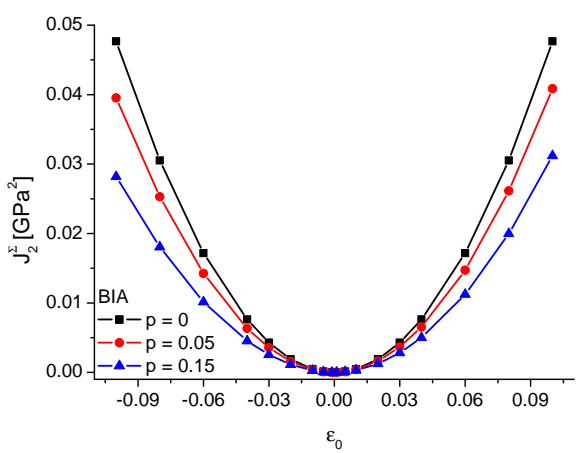

(d)

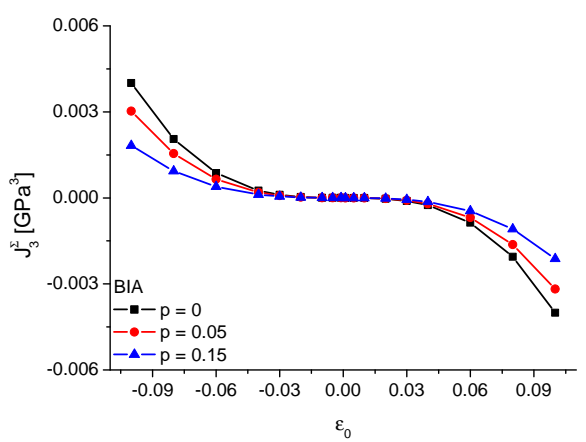

(f)

Figure 5: Macroscopic mechanical response of a bimodular single-pore rve (PL1, see Table 11 for different values of porosity $p$ and for uniaxial and biaxial loading scenarios. The case $p=0$ describes the bulk material behavior. 


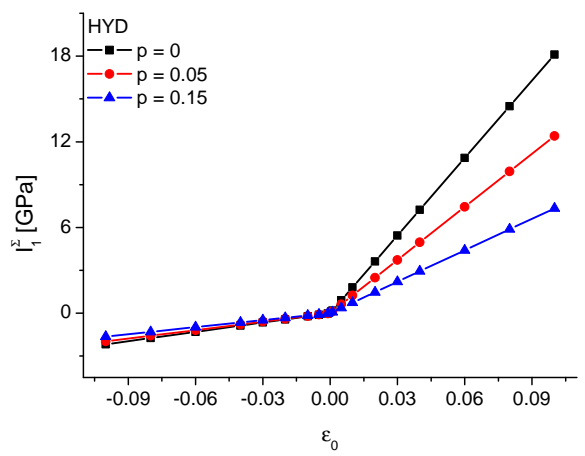

(a)

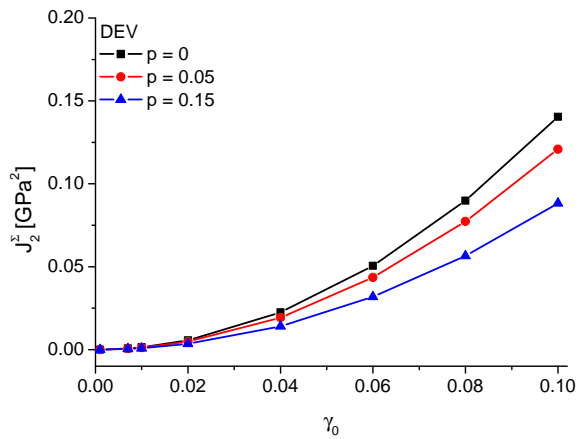

(c)

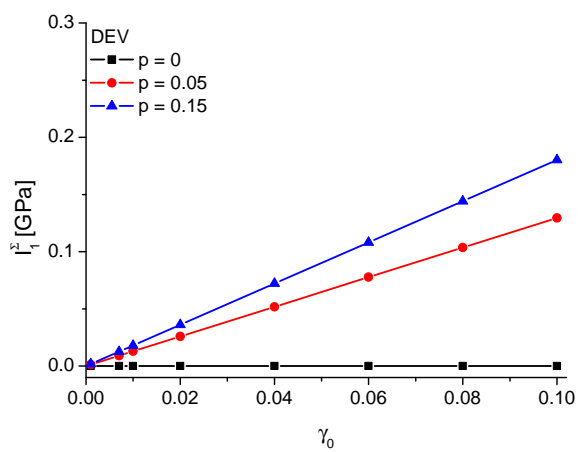

(b)

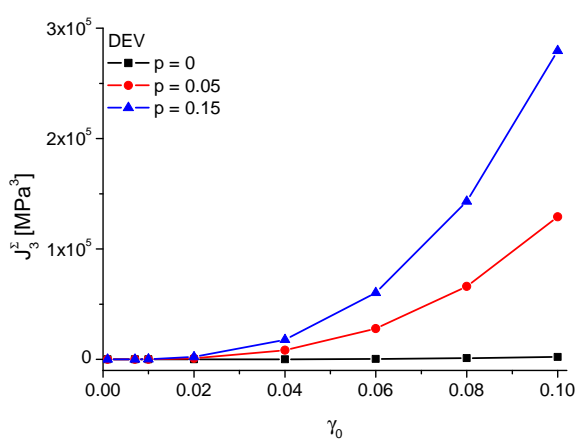

(d)

Figure 6: Macroscopic mechanical response of a bimodular single-pore rve (PL1, see Table 1 for different values of porosity $p$ and for hydrostatic and purely deviatoric loading scenarios. The case $p=0$ describes the bulk material behavior. 


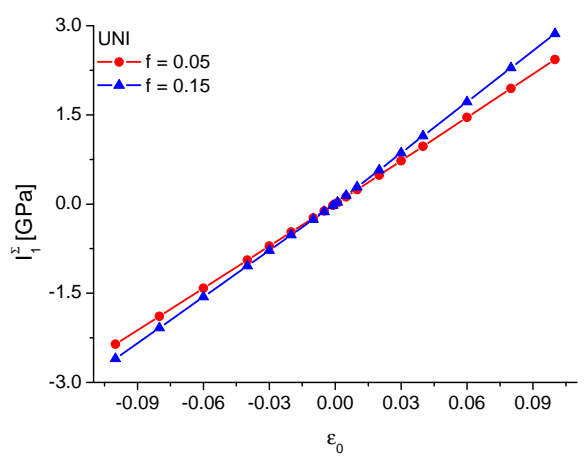

(a)

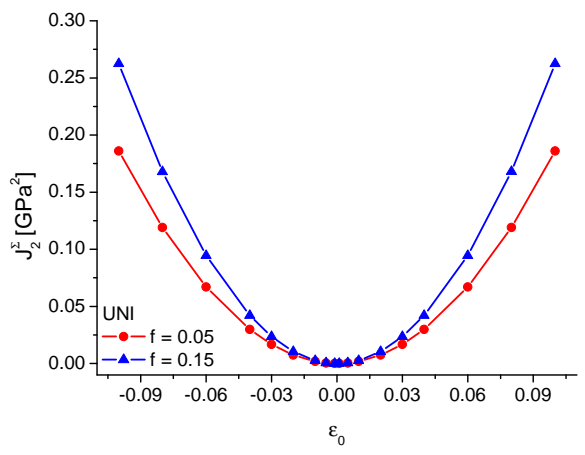

(c)

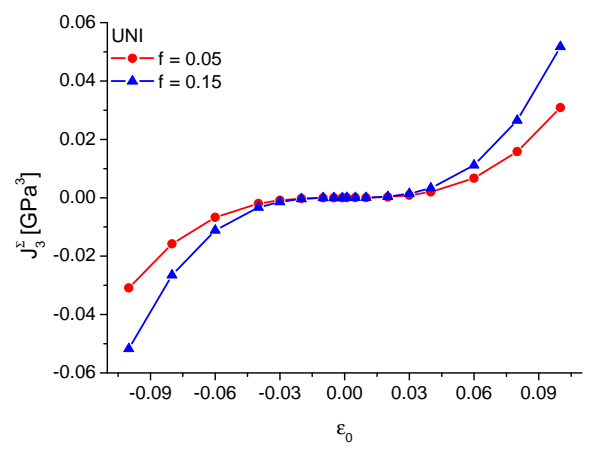

(e)

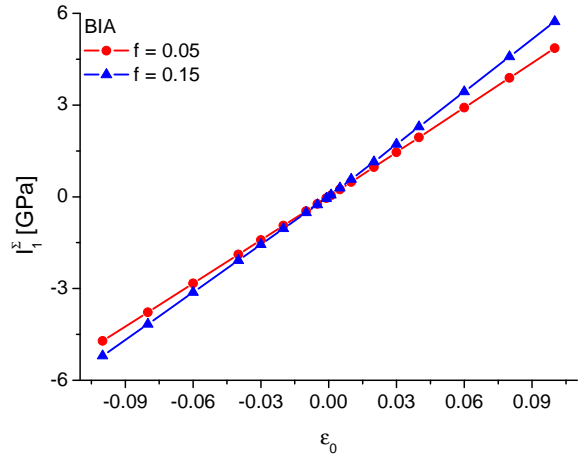

(b)

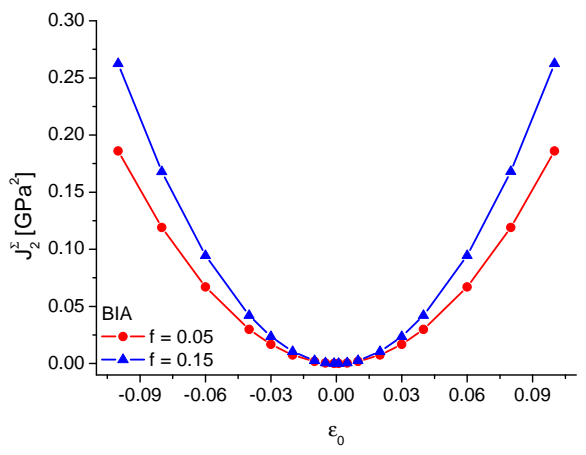

(d)

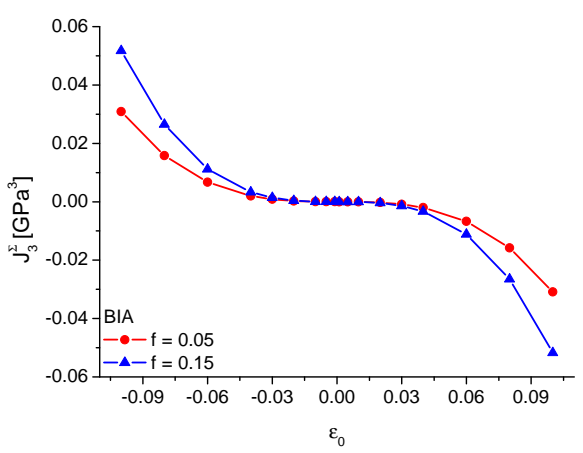

(f)

Figure 7: Macroscopic mechanical response of a single-inclusion rve, characterized by a linear matrix and a bimodular inclusion (L1_PL2, see Table 1), for different values of inclusion volume fraction $f$ and for uniaxial and biaxial loading scenarios. 


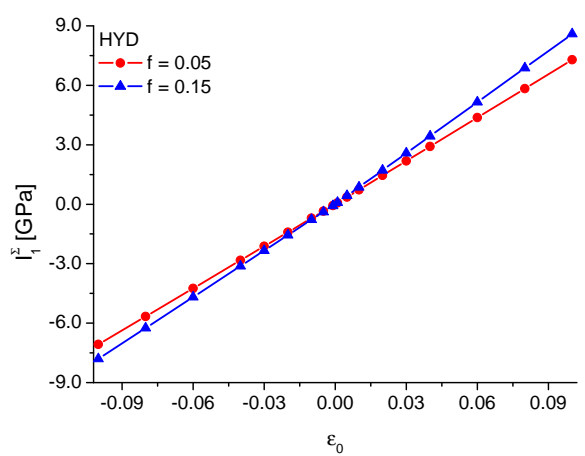

(a)

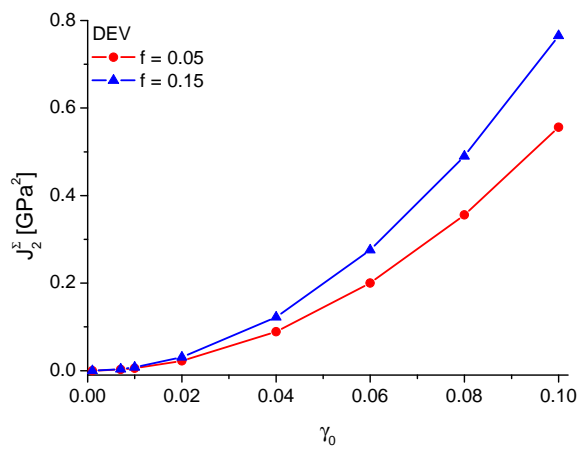

(c)

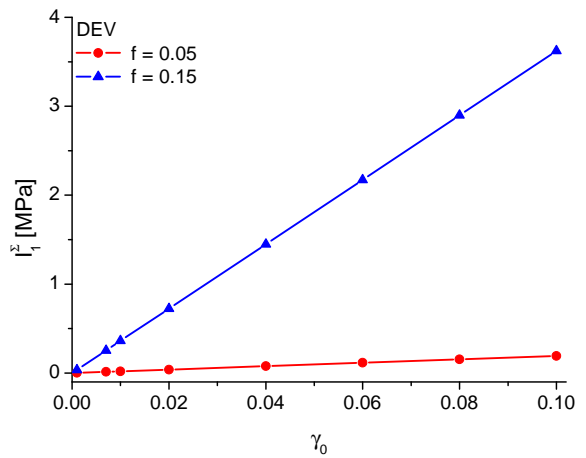

(b)

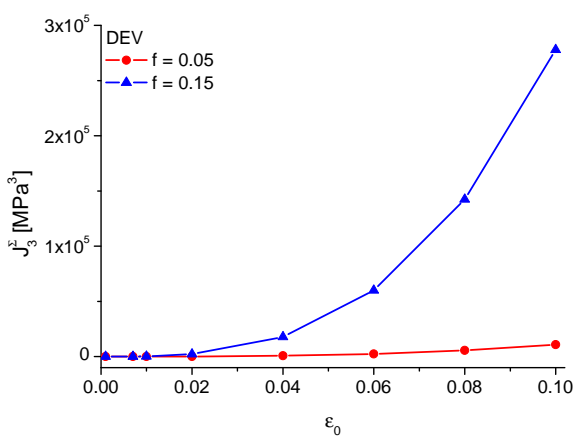

(d)

Figure 8: Macroscopic mechanical response of a single-inclusion rve, characterized by a linear matrix and a bimodular inclusion (L1_PL2, see Table 1), for different values of inclusion volume fraction $f$ and for hydrostatic and purely deviatoric loading scenarios. 
- the increase of the inclusion volume fraction $f$ induces an increase in both bilinear and asymmetry features;

- a purely deviatoric homogeneous strain boundary condition induces a coupling between hydrostatic and deviatoric mechanical responses, and this coupling increases with $f$ (see Fig. 10).

As the comparison between Figs. 9 10 and 11 12 confirms, a similar macroscale mechanical response is observed in the case PL1_PL2, that is by considering both matrix and inclusion as bimodular (Figs. 11 12 .

As previously recalled (see Section 2) a bimodular Curnier-type isotropic material is characterized by different bulk moduli in traction and compression and by a constant shear modulus. In this framework, present numerical results show that such a condition is no longer satisfied at the macroscale, the effective properties not complying with a bimodular Curnier-type isotropic behavior. Such an evidence is highlighted by the asymmetry depicted in panels (c) and (d) of Figs. 5. 9, and 12. Moreover, the occurrence of a significant coupling between hydrostatic and deviatoric macroscale responses (panels (b) and (d) of Figs. 6, 8, 10, and 12 suggests the onset of a certain anisotropy degree at the macroscale, strictly depending on the local perturbative effects induced by the pore or by the inclusion.

In order to furnish further insights on such an aspect, and since the observed similarities, in the following reference will be made only to the case PL1_L2 (linear elastic inclusion embedded in a bimodular matrix). In detail, Fig. 13 depicts the microscale distributions of the hydrostatic strain invariant $\left(I_{1}^{\varepsilon}\right)$, of the second-order deviatoric strain invariant $\left(J_{2}^{\varepsilon}\right)$, and of the bulk modulus $(k)$ computed under tension and compression biaxial loading conditions, for different inclusion volume fractions. Different patterns of the second-order deviatoric strain invariant are numerically experienced when tension or compression states are analyzed, confirming the asymmetry reported in Fig. 9(d) and resulting more evident by increasing $f$. Moreover, by referring to a purely deviatoric loading condition, Fig. 14 reports the distribution of the local bulk modulus as resulting by the sign of the microscopic hydrostatic strain. Depending on the inclusion volume fraction, the local constitutive response can be regarded as referred to three well-defined sub-phases, associated to the tension/compression bulk modulus restrictions and to the inclusion. Since the local distribution of the microscopic hydrostatic strain, corresponding to a null macroscopic hydrostatic strain value, a cross-shaped arrangement of tension/compression sub-phases can be distinguished within the matrix domain. In detail, the tension/compression sub-phases correspond to the $62 \% / 33 \%$ volume fractions of the overall domain for an inclusion volume fraction equal to $f=5 \%$, and to $52.7 \% / 32.3 \%$ volume fractions for $f=15 \%$. Such an intraphase heterogeneity has been computed as independent on the amount of the macroscopic deviatoric strain (i.e., on the value of $\gamma_{0}$ ).

Furthermore, the local distributions of the bulk modulus depicted in Fig. 14 give a straight indication on the source of the coupling effects. As a matter of fact, they derive from the loss in macroscopic constitutive symmetry induced by 
the previously-highlighted tension/compression cross-shaped arrangement, leading to the occurrence of a spherical macroscopic stress under a purely deviatoric macroscale strain state.

Such results have motivated the idea of considering the macroscopic mechanical response under mixed loading scenarios (HYD+DEV), defined by combining a macroscopic hydrostatic state (HYD) with a purely deviatoric one (DEV) (see Figs. 15 and 16). In this case, hydrostatic/deviatoric coupling effects produce a strong non-linear macroscopic response in the neighborhood of $\varepsilon_{0}=0$. In particular, a non linear transition from a compression-driven to a traction-driven behavior is observed for all considered stress invariants, with non-linear regimes increasing by improving the macroscopic deviatoric state. The volume fractions of tension/compression sub-phases change with the amount of the macroscopic hydrostatic loading (i.e., with $\varepsilon_{0}$ ). Such an occurrence is depicted in Fig. 17 that reports the evolution of the sub-phases volume fractions versus $\varepsilon_{0}$. As Fig. 16 shows, when $\varepsilon_{0}$ increases the localized intraphase heterogeneity and the corresponding loss of constitutive symmetry tend to disappear, leading to fully uncoupled tension-driven or compression-driven macroscopic behaviors. On the contrary, a suitable choice of the macroscopic spherical strain allows to control the heterogeneity distribution as well as the coupling effect.

\subsection{Multi-inclusion rves}

Numerical results obtained by considering PL1_L2 multi-inclusion rves are presented and analyzed. In these cases, since the random arrangements herein considered, the computed macroscopic mechanical response is practically equivalent to the one determined for the PL1_L2 single-inclusion rve (see Figs. 9] 10], with differences always less then $0.5 \%$. Thus, computations on multi-inclusion rves confirm that possible boundary effects arising on single-inclusion rves can be retained negligible. For the sake of compactness, macroscopic stress invariants computed for multi-inclusion rves are herein omitted.

Under a purely deviatoric loading condition, results in Figs. 18 and 19 depict the distributions of the local bulk modulus and of the microscopic hydrostatic strain. These results allow to confirm the occurrence of a cross-shaped arrangement of tension/compression sub-phases within the matrix domain and around each inclusion, as a result of local perturbative effects. Such an intraphase heterogeneity is associated to tension-driven and compression-driven matrix domains characterized by a well-defined average orientation (at about 45 degrees with respect $\mathbf{e}_{\mathrm{x}}$ and $\mathbf{e}_{\mathrm{y}}$ directions), confirming the onset of a certain loss of macroscopic constitutive symmetry degree. Although subphases align along clearly-visible mean directions, Figs. 18 and 19 highlight that both the local patterns of such matrix subphases and their coalescence are strictly depending on the inclusion arrangement and dimensions. In detail, small inclusions

tend to produce reduced intraphase heterogeneity and subphases coalescence is promoted when the inclusion distance is reduced. 


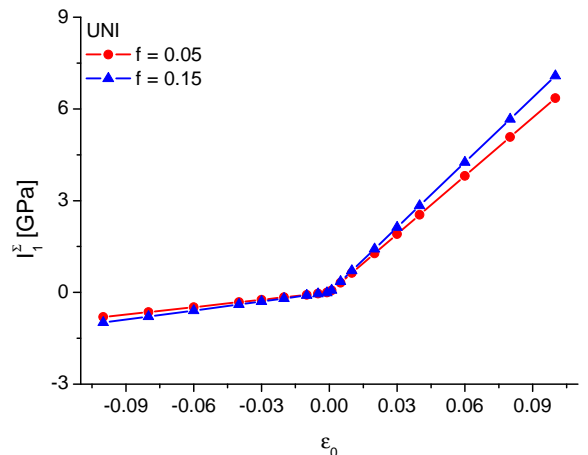

(a)

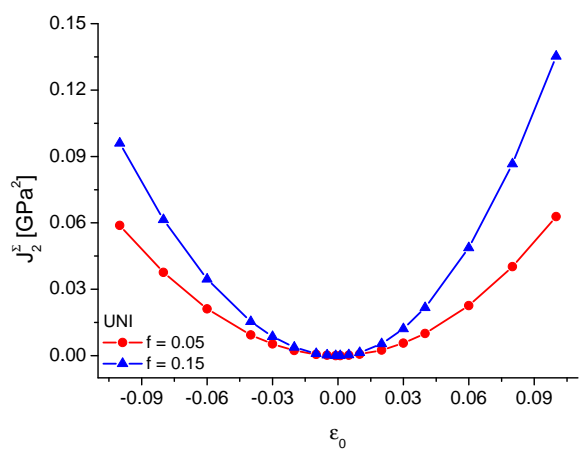

(c)

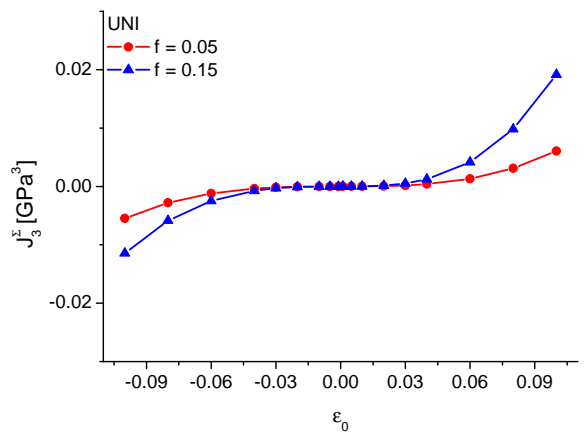

(e)

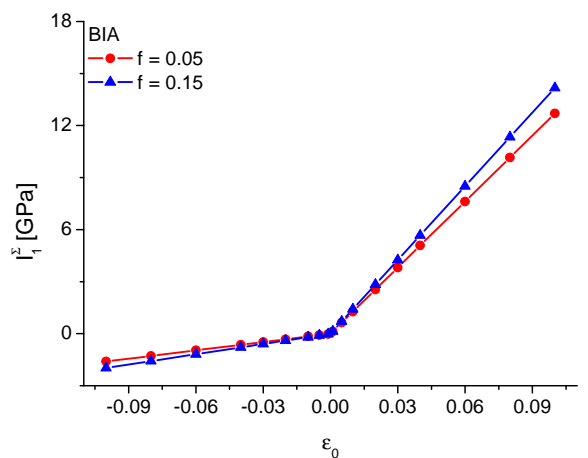

(b)

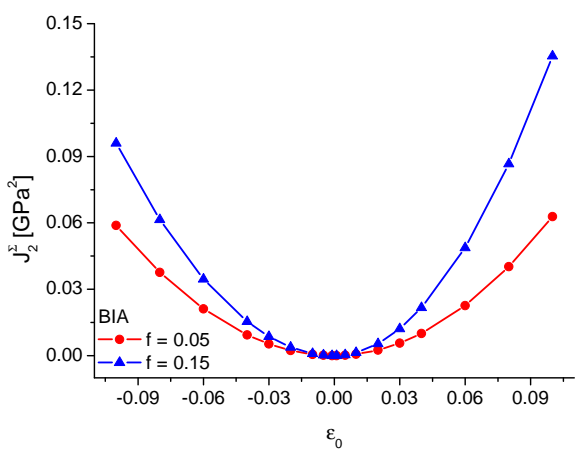

(d)

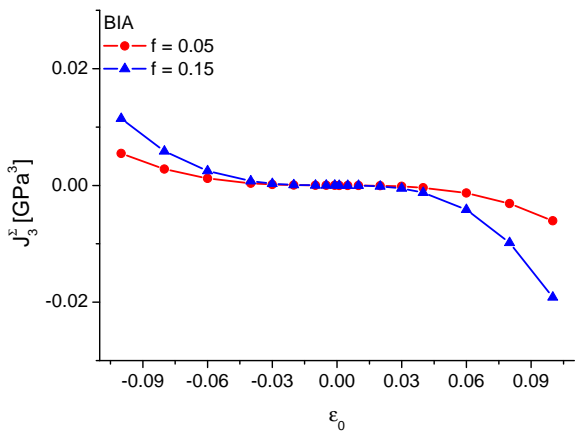

(f)

Figure 9: Macroscopic mechanical response of a single-inclusion rve, characterized by a bimodular matrix and a linear inclusion (PL1_L2, see Table 1), for different values of inclusion volume fraction $f$ and for uniaxial and biaxial loading scenarios. 


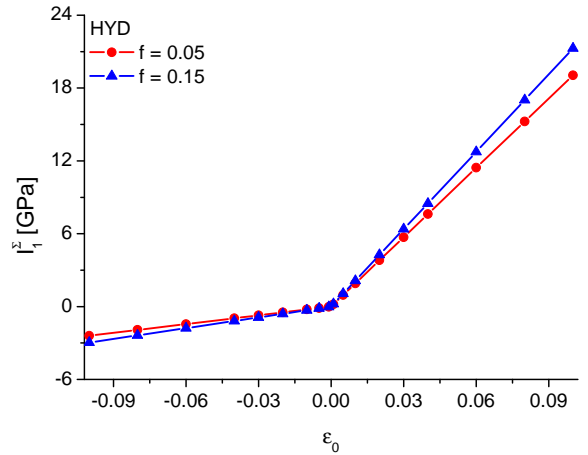

(a)

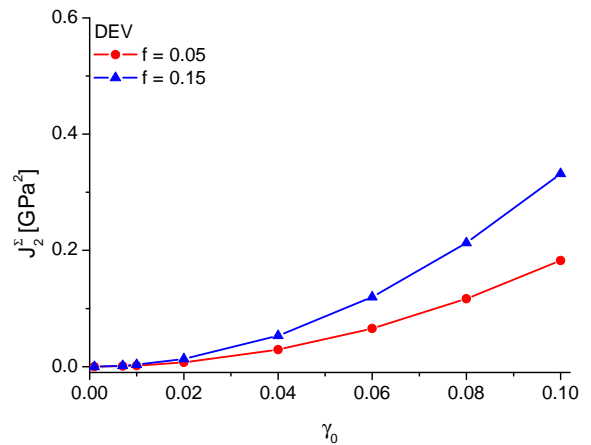

(c)

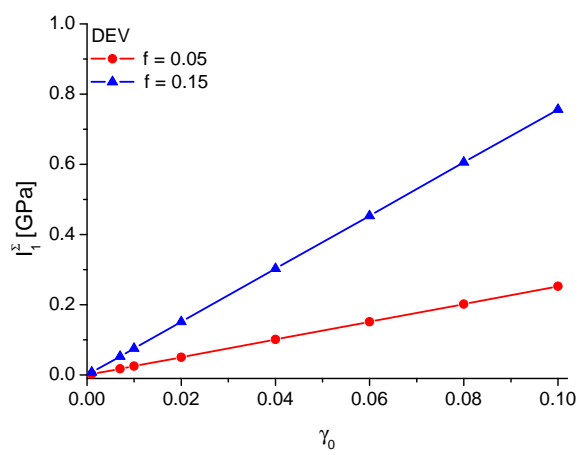

(b)

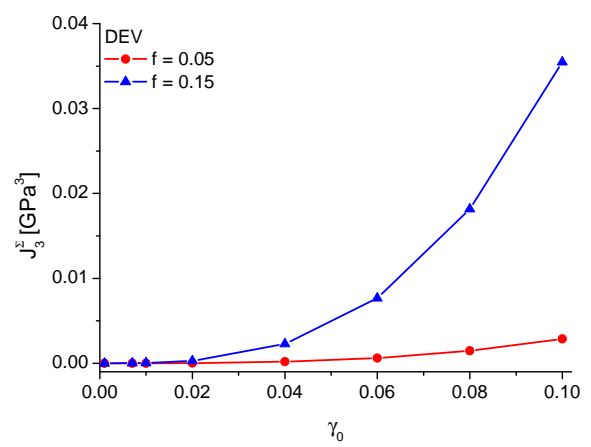

(d)

Figure 10: Macroscopic mechanical response of a single-inclusion rve, characterized by a bimodular matrix and a linear inclusion (PL1_L2, see Table 1), for different values of inclusion volume fraction $f$ and for hydrostatic and purely deviatoric loading scenarios. 


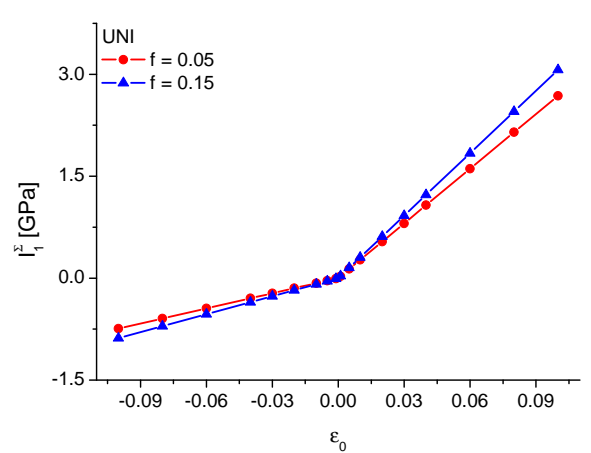

(a)

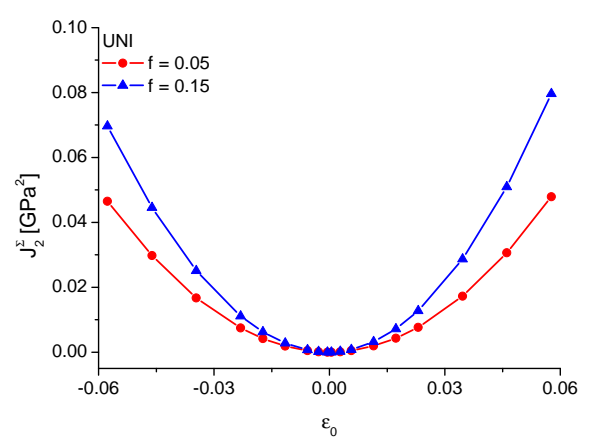

(c)

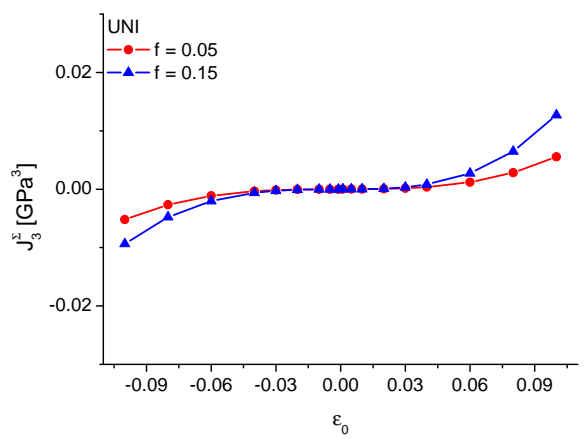

(e)

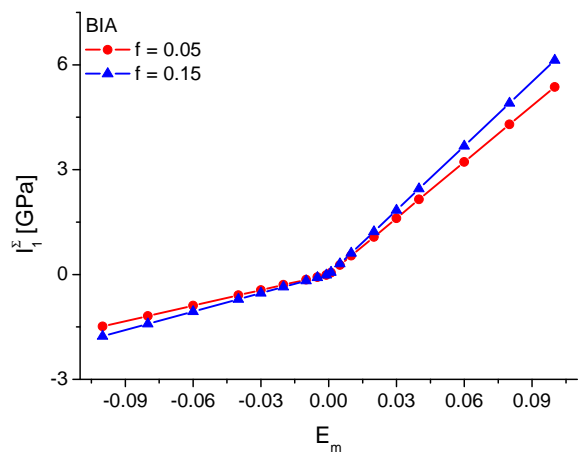

(b)

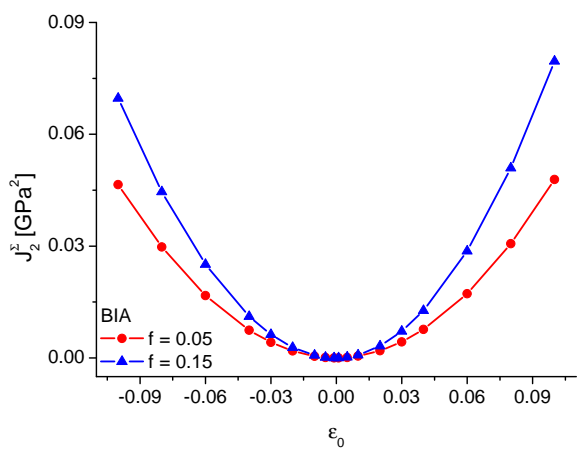

(d)

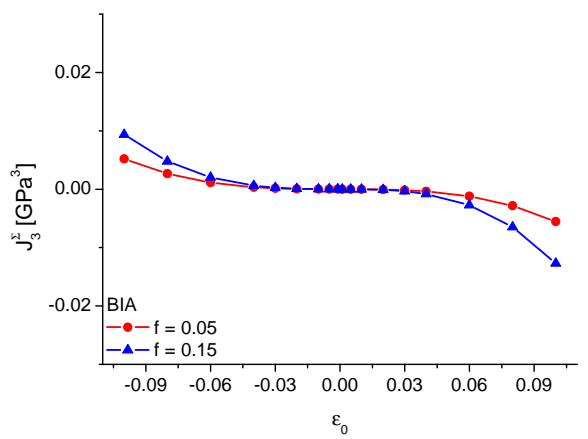

(f)

Figure 11: Macroscopic mechanical response of a single-inclusion rve, characterized by a bimodular matrix and a bimodular inclusion (PL1_PL2, see Table 1), for different values of inclusion volume fraction $f$ and for uniaxial and biaxial loading scenarios. 


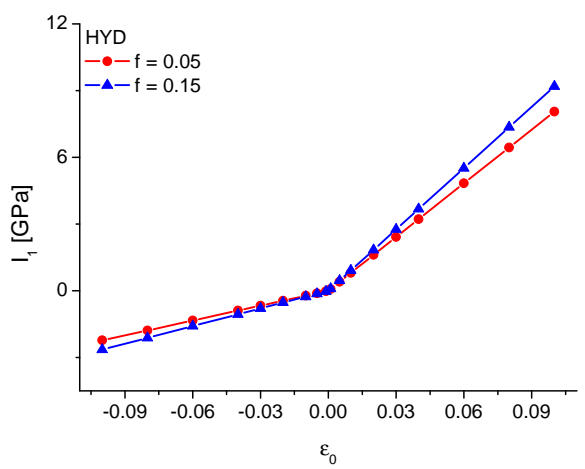

(a)

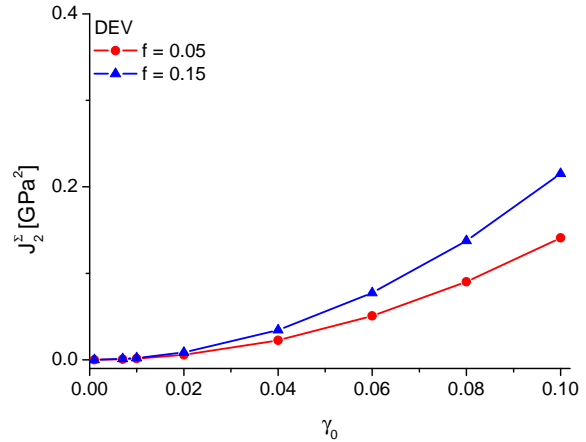

(c)

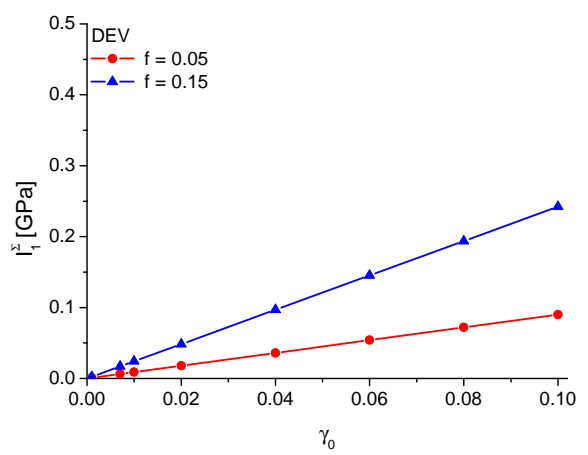

(b)

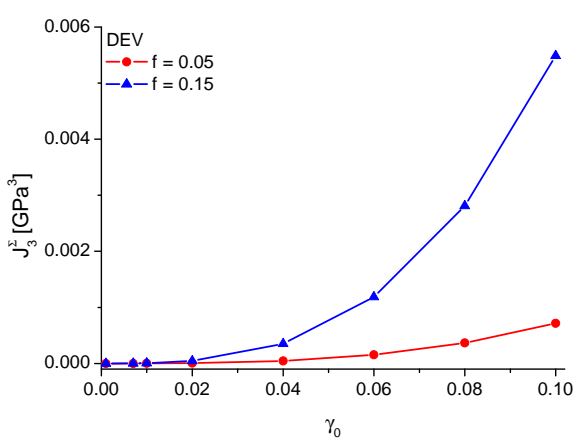

(d)

Figure 12: Macroscopic mechanical response of a single-inclusion rve, characterized by a bimodular matrix and a bimodular inclusion (PL1_PL2, see Table 1), for different values of inclusion volume fraction $f$ and for hydrostatic and purely deviatoric loading scenarios. 


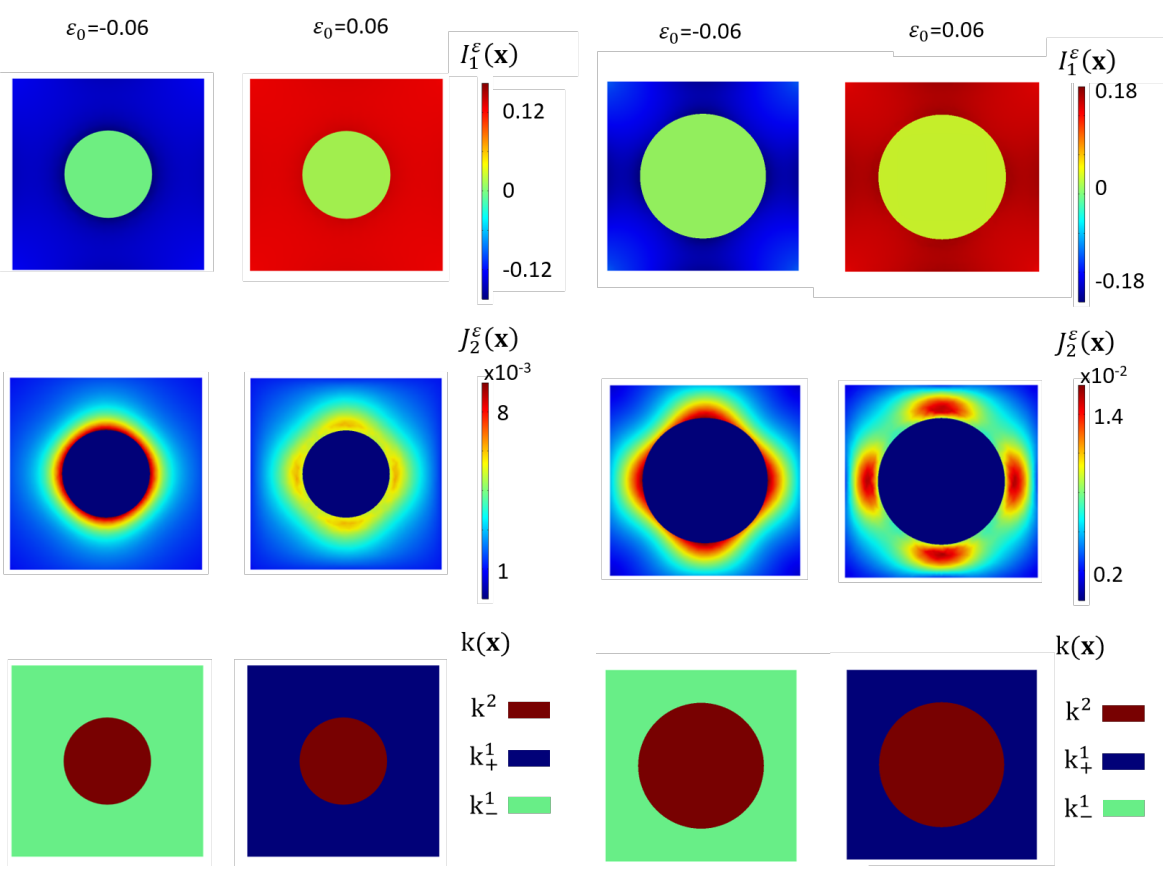

(a)

(b)

Figure 13: Case PL1_L2. Single-inclusion rve. Microscopic hydrostatic, and second-order deviatoric strain invariants, and bulk modulus on the $\pi$ plane (see Fig. 1), computed under tension and compression (left and right column, respectively) biaxial loading conditions. (a) $f=0.05 ;$ (b) $f=0.15$.
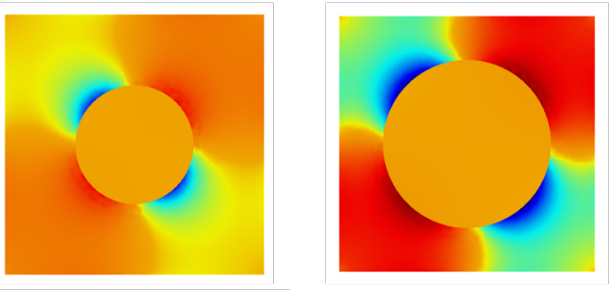

$I_{1}^{\varepsilon}(\mathbf{x})$
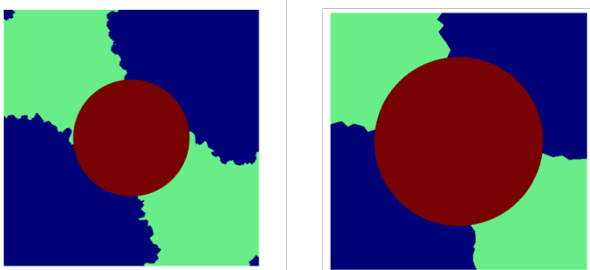

$\mathrm{k}(\mathbf{x})$

$\mathrm{k}^{2}$

$\mathrm{k}_{+}^{1}$

$\mathrm{k}_{-}^{1}$

Figure 14: Case PL1_L2. Single-inclusion rve. Microscopic hydrostatic strain invariant and bulk modulus on $\pi$ plane (see Fig. 11, computed under a purely deviatoric loading condition $\left(\gamma_{0}=0.06\right)$ for $f=0.05$ (left column) and $f=0.15$ (right column). 

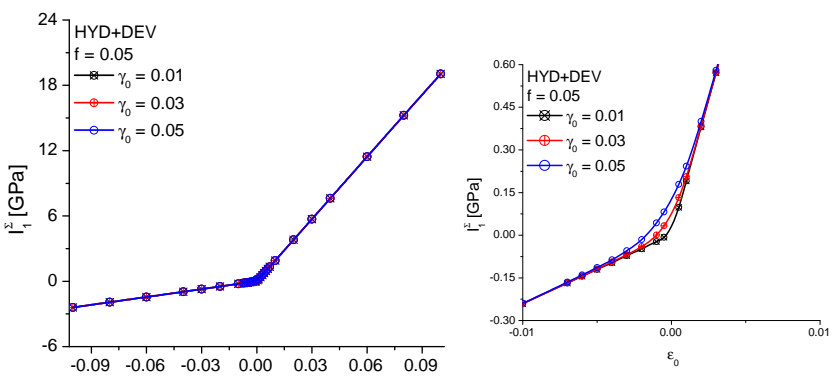

(a)
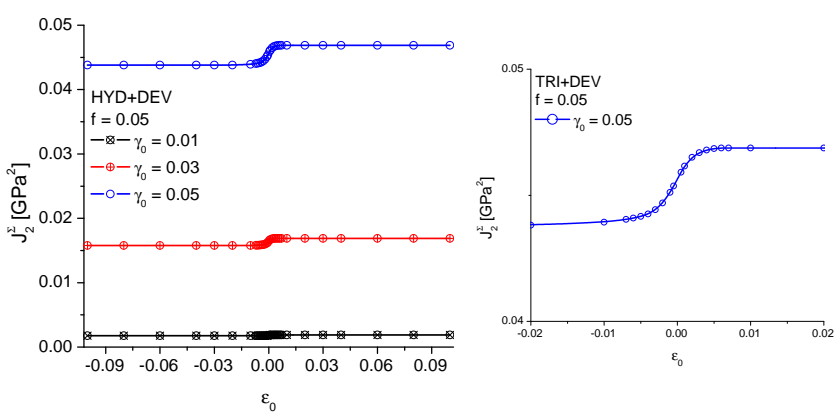

(b)
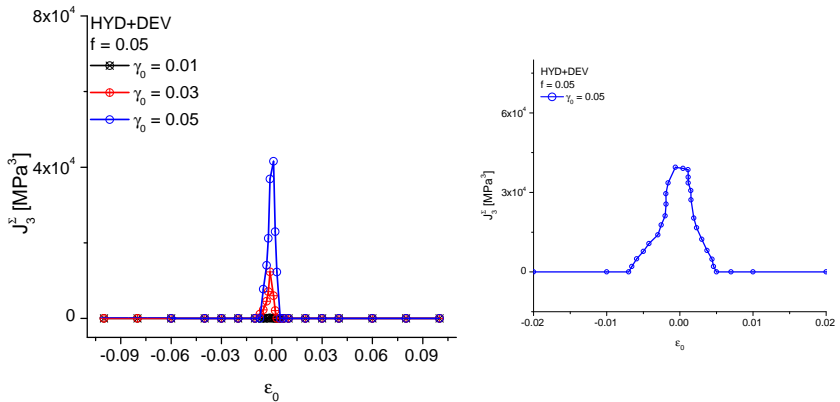

(c)

Figure 15: Macroscopic mechanical response of a single-inclusion rve, characterized by a bimodular matrix and a linear inclusion (PL1_L2, see Table11, for inclusion volume fraction $f=0.05$ and under a mixed load (HYD $+\mathrm{DEV})$, for different values of $\gamma_{0}$ and macroscopic spherical strain $\varepsilon_{0}$ (see Eq. (5)). On the right, details of the non-linear regimes in the neighborhood of $\varepsilon_{0}=0$ are reported. 

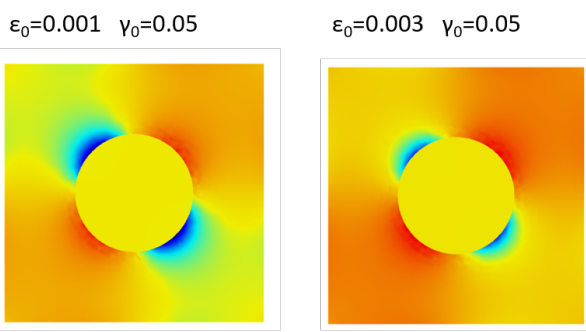

$\varepsilon_{0}=0.007 \quad \gamma_{0}=0.05$
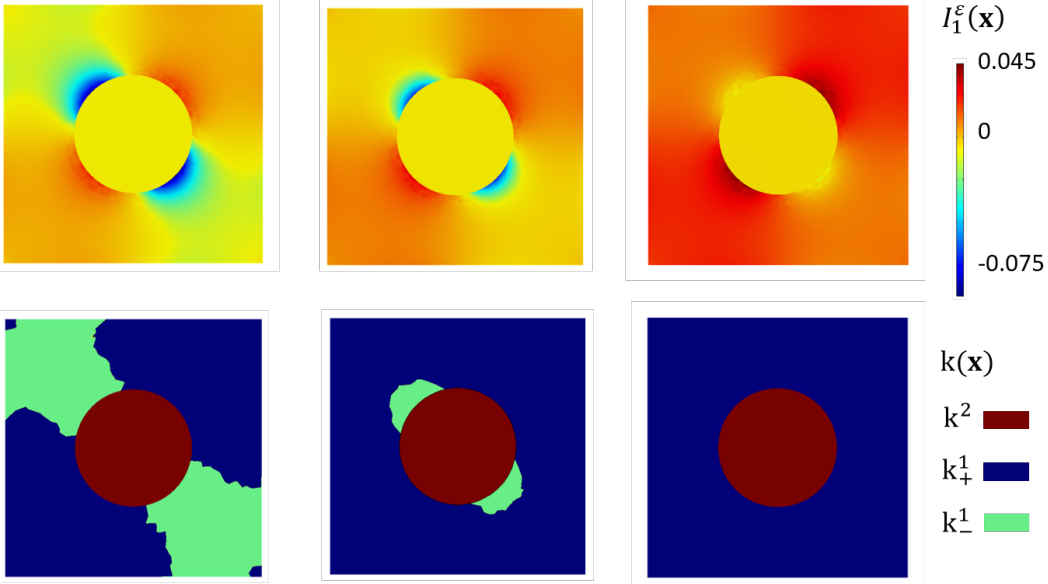

$\mathrm{k}(\mathbf{x})$

$\mathrm{k}^{2}$

$\mathrm{k}_{+}^{1}$

$\mathrm{k}_{-}^{1}$

Figure 16: Case PL1_L2. Single-inclusion rve. Microscopic hydrostatic strain invariant and bulk modulus on $\pi$ plane (see Fig. 1) computed under a mixed loading condition $(\mathrm{HYD}+\mathrm{DEV})$, for $f=0.05$ and for different values of $\varepsilon_{0}$.

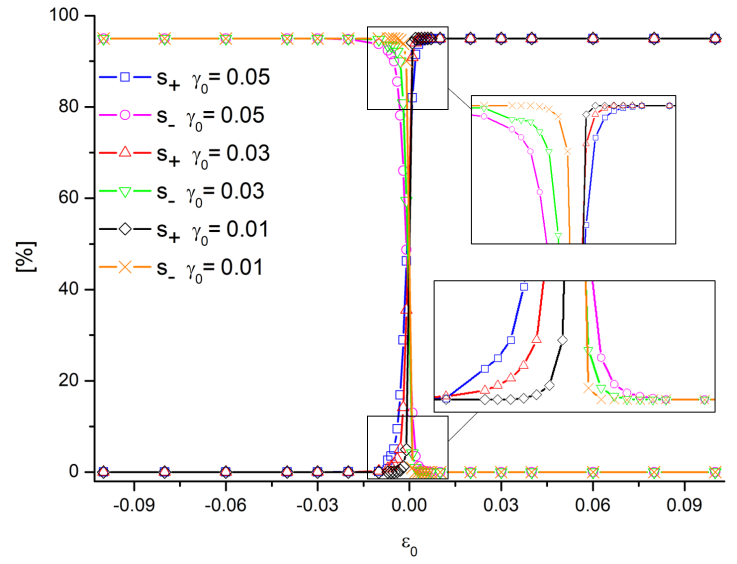

Figure 17: Case PL1_L2. Single-inclusion rve. Evolution of volume fractions for the tension and compression sub-phases, computed under a mixed loading condition (HYD+DEV) and for $f=5 \%$, versus $\varepsilon_{0}$. Volume fractions of the tension and the compression sub-phases are denoted with $s_{+}$and $s_{-}$, respectively. 

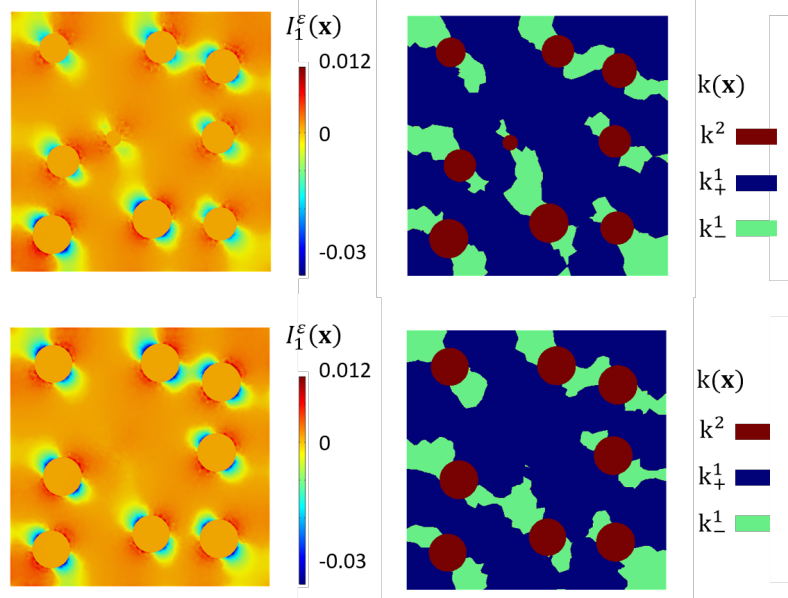

$\mathrm{e}_{\mathrm{y}}$ $e_{x}$

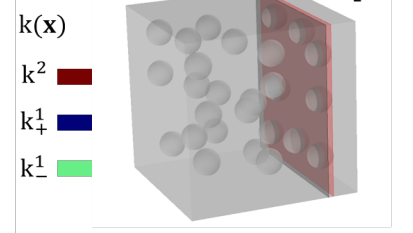

Figure 18: Multi-inclusion randomically arranged rve (PL1_L2, $f=0.05,27$ identical inclusions). Microscopic hydrostatic strain invariant and bulk modulus distribution computed under a purely deviatoric loading condition $\left(\gamma_{0}=0.02\right)$ on two different rve cross-sections (indicated in red in the bottom side of the Figure).
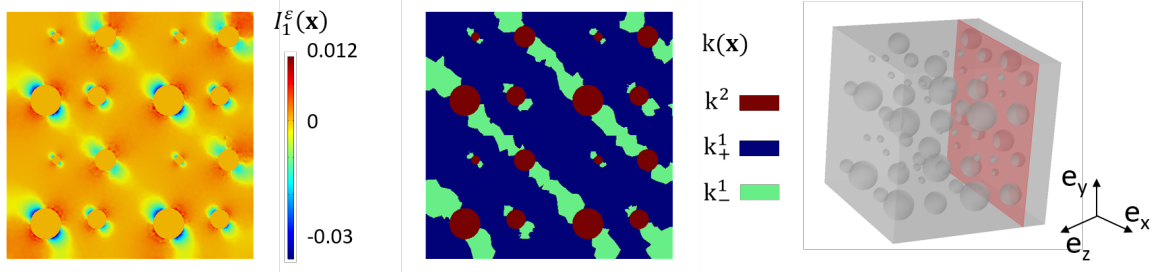

Figure 19: Multi-inclusion randomically arranged rve (PL1_L2, $f=0.05,64$ inclusions with different sizes). Microscopic hydrostatic strain invariant and bulk modulus distribution computed under a purely deviatoric loading condition $\left(\gamma_{0}=0.02\right)$ on the rve cross-section indicated in red. 


\section{Conclusions}

In this paper, the non-linear mechanical response of heterogeneous materials comprising bimodular phases is numerically investigated via a computational strategy based on an iterative finite-element scheme. Elastic bimodularity is dealt with reference to the theoretical framework developed by ?. Porous, singleand multi-inclusion models undergoing different homogeneous strain boundary conditions are considered, by combining matrix and/or inclusion bimodularity descriptions with a linearly elastic model.

As recalled in Section 2 an isotropic bimodular Curnier-type material is characterized by different bulk moduli in tension and compression and by a constant shear modulus. In this framework, experienced numerical results have shown that at the macroscale such a requirement is no longer satisfied for the cases under investigation. In detail, although the effective material response is characterized by a bilinear elastic behavior, the heterogeneous material does not behave as an isotropic bimodular Curnier-type matter. In order to investigate about the possibility that effective material response can comply with a more complex bilinear response (i.e., anisotropic bimodular Curnier type) different macroscale strain-based conditions should be considered for defining neutral states. Nevertheless, this is beyond the purpose of the present work and it will be addressed in future studies.

Proposed results highlighted that, when the matrix is bimodular (for both porous and composite rves), the occurrence of a significant coupling between hydrostatic and deviatoric macroscale responses is observed. Such coupling effects suggest the onset of a certain anisotropy degree at the macroscale, strictly depending on the local perturbation on the microscale strain field induced by the pore or by the inclusion. A one-way coupling mechanism is also observed: purely macroscopic hydrostatic strain-based condition does not introduce any deviatoric stress effect at the macroscale, whereas a purely deviatoric strain state produces effective hydrostatic stresses. Moreover, under a purely deviatoric loading condition, the analysis of the local bulk modulus distribution, as result of the sign of the microscopic hydrostatic strain, has confirmed the occurrence of a cross-shaped arrangement of traction/compression sub-phases within the matrix

domain for both single-inclusion and multi-inclusion rves. Hence, the macroscale costitutive response changes depending on the combination of hydrostatic and deviatoric loading.

\section{Appendix}

In what follows, the theoretical background of the elastic bimodularity developed by ? is briefly recalled. Bimodular materials are herein assumed to be described via a piece-wise linearly elastic response, representing a subclass of the so-called conewise linear elastic materials.

Let the local strain state $\varepsilon$ belong to the strain space $\mathcal{E}$. The latter is assumed to be divided into two complementary subdomains, by means of the 
hypersurface $\mathcal{I}$ described by the equation $g(\varepsilon)=0$, with $g$ being a continuous differentiable scalar function of $\varepsilon$. Therefore, it results $\mathcal{E}=\mathcal{E}_{-} \cup \mathcal{E}_{+} \cup \mathcal{I}$, where

$$
\begin{aligned}
& \mathcal{I}=\{\varepsilon \in \mathcal{E} \mid g(\varepsilon)=0\} \\
& \mathcal{E}_{-}=\{\varepsilon \in \mathcal{E} \mid g(\varepsilon)<0\} \\
& \mathcal{E}_{+}=\{\varepsilon \in \mathcal{E} \mid g(\varepsilon)>0\}
\end{aligned}
$$

Accordingly, by assuming the condition $g(\varepsilon)=0$ as representative of a strainbased neutral state, $\mathcal{E}_{-}$and $\mathcal{E}_{+}$can be thought as corresponding to contiguous strain subdomains associated to different stiffness tensors.

Correspondingly, the piece-wise linear stress-strain relationship straight results in:

$$
\boldsymbol{\sigma}(\varepsilon)=\mathbb{C}(\varepsilon): \varepsilon= \begin{cases}\boldsymbol{\sigma}_{-}(\varepsilon)=\mathbb{C}_{-}: \boldsymbol{\varepsilon} & \text { if } g(\varepsilon) \leq 0 \\ \boldsymbol{\sigma}_{+}(\varepsilon)=\mathbb{C}_{+}: \varepsilon & \text { if } g(\varepsilon) \geq 0\end{cases}
$$

where $\mathbb{C}_{-}$and $\mathbb{C}_{+}$identify compression-like and tensile-like fourth-order elasticity tensors, respectively, satisfying major and minor symmetries.

Continuity requirements at $\mathcal{I}$ for both strain energy and second-order stress tensor $\boldsymbol{\sigma}$ imply (?) that:

$$
[\mathbb{C}(\varepsilon)]:=\mathbb{C}_{+}-\mathbb{C}_{-}=s \frac{\partial g(\varepsilon)}{\partial \varepsilon} \otimes \frac{\partial g(\varepsilon)}{\partial \varepsilon} \quad \forall \varepsilon \mid g(\varepsilon)=0
$$

where $s$ is the jump coefficient and $\partial g(\varepsilon) /\left.\partial \varepsilon\right|_{\mathcal{I}}$ denotes the unit normal at the hypersurface $\mathcal{I}$. As formally proved in ?, since $\mathbb{C}_{+}$and $\mathbb{C}_{-}$are constant, the compression/tension interface $\mathcal{I}$ reduces to a hyperplane. Moreover, relationship (8) prescribes that the elasticity-tensor jump across $\mathcal{I}$ is normal to the interface and there is no tangential discontinuity.

It is worth pointing out that the elasticity tensor $\mathbb{C}$ in Eq. (7) does not exist in a classical sense at the interface $\mathcal{I}$. Nevertheless, by excluding inextensible and incompressible materials, it can be represented through the concepts of subgradient and subdifferential (?).

Assuming that the constitutive restrictions in Eq. (7) obey to isotropic symmetry, it is possible to show that (?):

- the interface $\mathcal{I}$ is defined by the hyperplane $g(\varepsilon)=\operatorname{tr}(\varepsilon)=0$, whose unit normal is the second-order identity tensor $\mathbf{I}=\partial \operatorname{tr}(\varepsilon) / \partial \varepsilon$,

- the bulk modulus $k$ is piece-wise constant with a jump for $\operatorname{tr}(\varepsilon)=0$,

- the shear modulus $\mu$ is constant (namely, continuous through $\mathcal{I}$ ).

Therefore, the elasticity tensor can be written as:

$$
\mathbb{C}(\varepsilon)=\left\{\begin{array}{rlrl}
\mathbb{C}_{-}=2 \mu \mathbb{K}+3 k_{-} \mathbb{J} & & \text { if } \operatorname{tr}(\varepsilon)<0 \\
\mathbb{C}_{+}=2 \mu \mathbb{K}+3 k_{+} \mathbb{J} & & \text { if } \operatorname{tr}(\varepsilon)>0 \\
\partial_{\varepsilon} \boldsymbol{\sigma} & =\mathbb{C}_{-}+3(1-\lambda) s \mathbb{J} & & \text { if } \operatorname{tr}(\varepsilon)=0 \\
& =\mathbb{C}_{+}-3 \lambda s \mathbb{J} \quad \forall \lambda \in[0,1] &
\end{array}\right.
$$


where $\partial_{\varepsilon} \boldsymbol{\sigma}$ is the set of subgradients of the stress field with respect to the strain, $\mathbb{J}=(\mathbf{I} \otimes \mathbf{I}) / 3$ and $\mathbb{K}=\mathbb{I}-\mathbb{J}$ are the hydrostatic and deviatoric fourth-order projector tensors ( $\mathbb{I}$ being the fourth-order identity tensor), respectively (?), and

$k_{-}$and $k_{+}$are the compression/tension restrictions of the bulk modulus, with $s=[k(\varepsilon)]=k_{+}-k_{-}$when $\operatorname{tr}(\varepsilon)=0$.

\section{References}

